\title{
DETERMINATION OF CHARGE AND SIZE OF RINGS BY ATOMIC FORCE MICROSCOPY
}

\author{
Daniel Lazarev and Fredy R. Zypman ${ }^{1}$ \\ Yeshiva University, Department of Physics, New York, NY 10033, USA
}

\begin{abstract}
We present and solve the problem of measuring charge and size of a ring from electrostatic forces in Atomic Force Microscopy (AFM). We focus our attention on the charge-induced-charge force. From the electric field, the AFM-ring forceseparation curve is computed and, based on the shape of the force-separation curve, an algorithm is proposed based on measuring the maximum experimental force to invert the problem. Thus the novelty of this paper lays in the calculation of the ring-AFM-tip force-separation curve and in the identification of features of the curve useful to experimentally measure the charge and size of the ring.
\end{abstract}

Keywords. Atomic Force Microscopy, Electrostatic Force Microscopy, Charged Ring, Scanning Force Spectroscopy, Nanoscale Charge Measurement.

\section{INTRODUCTION}

We consider a charged ring probed by the tip sensor of an Atomic Force Microscope (AFM) and present a method to measure, from the force-vs-separation curve, the charge and size of the ring.

A number of systems of nano- or sub-nanometer dimensions can be modeled as rings of charge. For example in molecular pumps, rings are driven, based on their charge content, to link amino acids into growing peptides. Knowledge of charge content

${ }^{1}$ Corresponding author. zypman@yu.edu 
and size is necessary to apply these pumps to growing other assembles. ${ }^{1}$ More generally, charge plays a key role in the structure attained by large molecules when they self-assemble. ${ }^{2}$ There have also been proposals to use charged rings to physically store qubits for quantum computing. ${ }^{3}$ These and other examples underscore the relevance of understanding electrostatics measurements at the nanoscale with the AFM.

In this paper, we first compute the force vs separation curve of electrostatic interaction between a charged ring and an AFM tip. Then, by focusing on the separation and value of the maximum force-a simple practical features to identify in an experiment-two equations are obtained that permit the measurement of both the ring size and charge.

\section{THEORY}

The arrangement of the system is depicted in Figure 1, and should help the interested reader to reproduce the results presented here. A ring of radius A and uniform charge density $\lambda$ lays on an insulating substrate. The tip of the AFM is modeled as a sphere of radius $R$. One on hand, this is a reasonable assumption at the nanometer length scales relevant to the problem since the pyramidal tip becomes blunt at these length scales., $4,5,6,7$ On the other hand, there are explicitly spherical tips commonly used in AFM, both dielectric and metallic. ${ }^{8}$ Here we consider the common type of metal or metallically coated tip. ${ }^{9}$ 


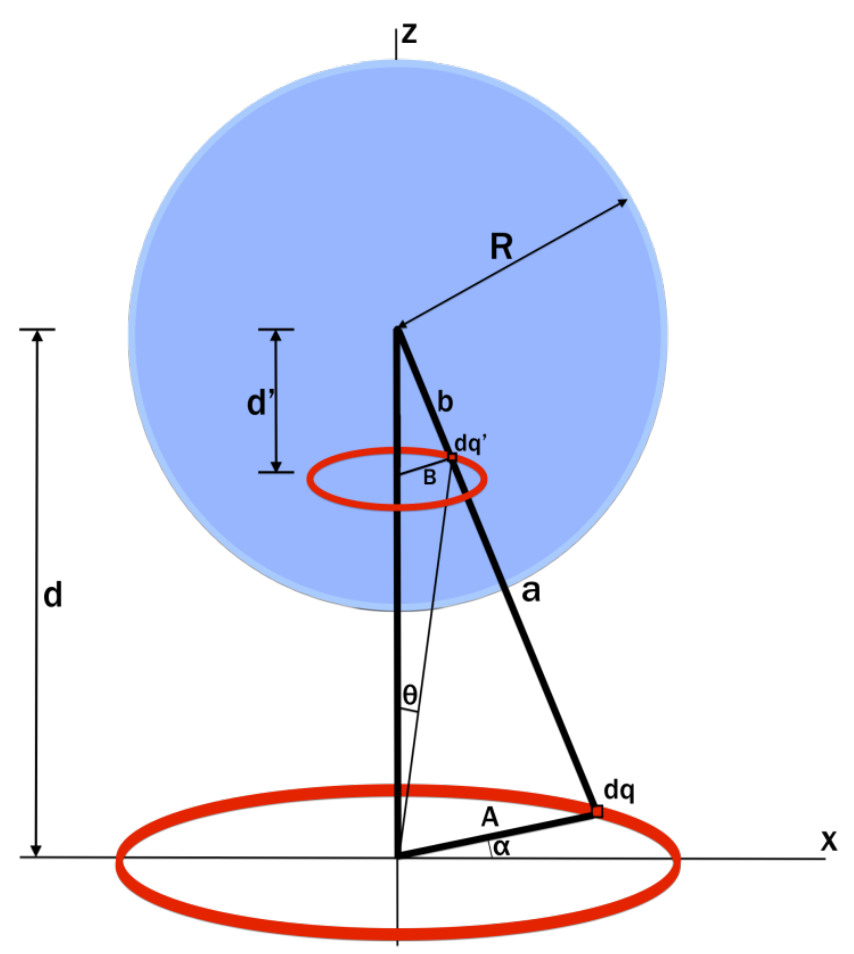

Figure 1. A charged ring lays on a horizontal insulating substrate. An AFM conducting tip hovers at a height $d$ above the plane. The tip is attached from the top to a cantilever (not shown).

Our goal is to provide an algorithm that allows to measure the ring radius $\mathrm{A}$ and its charge density $\lambda$ from the AFM direct experimental forces. To this end, we first calculate the electrostatic attractive force between the ring and the tip. The force is calculated by using the method of images ${ }^{10}$ (which encapsulates the polarization of the tip) as follows (refer to Figure 1). We first find the locus of the image of the ring by the inversion with respect to the sphere which in this case of axial symmetry is also a ring but of radius $B=\frac{A R^{2}}{A^{2}+d^{2}}$, away from the center of the sphere a distance $d^{\prime}=\frac{R^{2}}{A^{2}+d^{2}} d$. The element of charge is proportional to $d q$, explicitly $d q^{\prime}=-\frac{R}{\sqrt{A^{2}+d^{2}}} d q$. Hence the force is obtained by first evaluating the $\mathrm{z}$ component of the electric field produced by the ring at the location of $d q^{\prime}$, multiplying by $d q^{\prime}$ to obtain the force 
component differential $d F_{z}$, and finally integrating around the ring angular coordinate $\alpha$. It is to be noted that, even for case not axially symmetric, the major force measured in AFM is in the $\mathrm{z}$ direction since the system's motion is constrained along $\mathrm{x}$ and $\mathrm{y}$.

We now focus on the details that allow an explicit calculation of the vertical force. We follow the notation introduced in our previous work on rings of charge ${ }^{13}$; forces between rings of charge have been reported. ${ }^{11,12}$ In the calculation that follows, it must be appreciated that it is not the force between two prescribed rings that we are computing. Rather it is the force between one prescribed ring, and a second one that evolves (in size, location, and charge content) as the tip-sample separation changes, thus representing the charge/induced-charge interaction. This is a new result and conceptually relevant since the separation dependence of the force between two prearranged charged objects is different from the force between a given charged object and its induced charge. As a simple example, notice that the Coulomb force between two given charges decays as the square of their distance, while the force between a charge and its induced charge on a sphere decays as the cube of their distance at large separations.

We take the origin of coordinates at the center of the ring, and the $\mathrm{z}$ axis perpendicular to the plane of the ring (see Figure 2). 


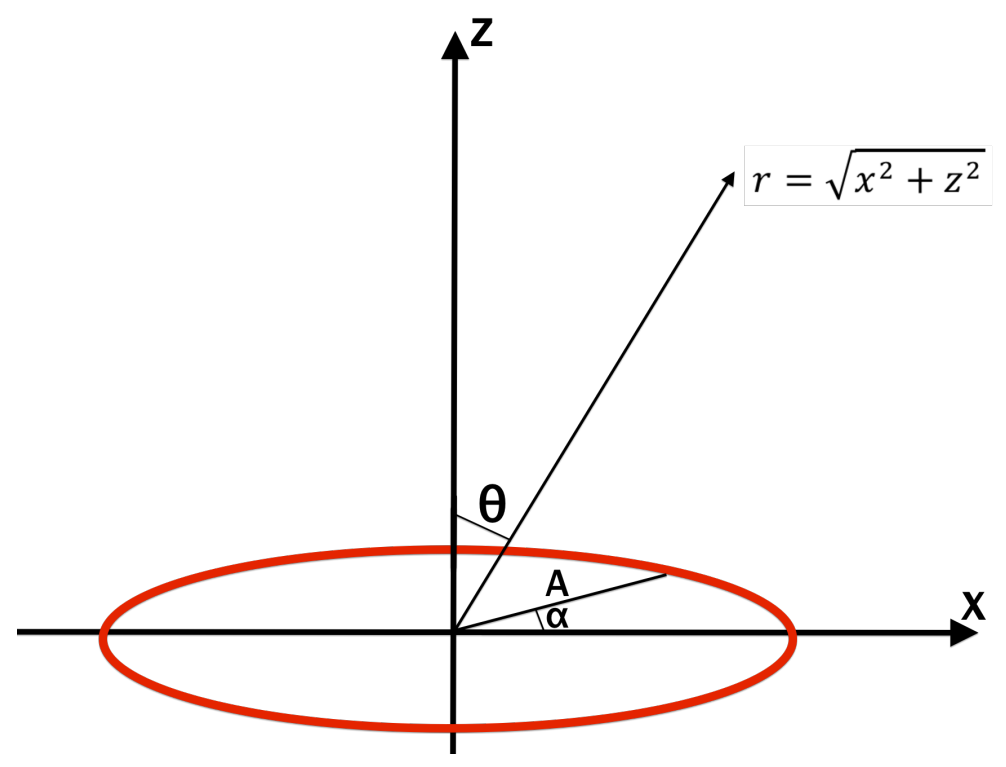

Figure 2. Single charged ring and parameters that specify the evaluation point of the electric field.

The electric field produced by a ring of charge is known throughout the whole space, including outside the symmetry axis. ${ }^{13}$ For this work we only need the zcomponent,

$$
E_{z}(\xi, \theta)=\frac{\lambda}{2 \pi \varepsilon_{0} A} \frac{\xi \cos \theta}{\left(\xi^{2}+1\right)^{\frac{3}{2}}} f(\mu)
$$

where $\mu=\frac{2 \xi}{\xi^{2}+1} \sin \theta, \xi=\frac{\sqrt{z^{2}+x^{2}}}{A}$ is the distance from the center of the ring in units of A, $f(\mu)=\int_{0}^{\pi} \frac{d \alpha}{(1-\mu \cos \alpha)^{\frac{3}{2}}}=-\frac{2 \mathbb{E}\left(\frac{2 \mu}{1+\mu}\right)}{(\mu-1) \sqrt{1+\mu}}$, with $\mathbb{E}(m)$ the Elliptic Integral of the Second Kind $^{14}$ and $\theta$ is the angle of the observation point vector with respect to the $z$-axis. Integrating with respect to $\alpha$, we arrive at an expression between the ring and the AFM tip:

$$
F_{z}(\eta, \beta)=-F_{0} \frac{\eta \beta^{2}}{\sqrt{\beta^{2}+\eta^{2}}}\left\{\frac{1-\frac{1}{\beta^{2}+\eta^{2}}}{\left[\beta^{2}+\left(\frac{\beta}{\beta^{2}+\eta^{2}}\right)^{2}+\left(\eta-\frac{\eta}{\beta^{2}+\eta^{2}}\right)^{2}\right]^{\frac{3}{2}}}\right\} f(\mu)
$$


where we have substituted $F_{0} \equiv \frac{\lambda^{2}}{\varepsilon_{0}}$ as the basic unit of force, and where $\mu$ defined above can be written explicitly in terms of the experimental parameters,

$$
\mu=\frac{\frac{2 \beta^{2}}{\beta^{2}+\eta^{2}}}{\left(\frac{\beta}{\beta^{2}+\eta^{2}}\right)^{2}+\left(\eta-\frac{\eta}{\beta^{2}+\eta^{2}}\right)^{2}+\beta^{2}}
$$

Also $\eta \equiv \frac{d}{R}$ is the separation between the tip and the plane of the ring and $\beta \equiv \frac{A}{R}$ is the radius of the ring, both in units of the radius of curvature of the tip. The radius of curvature of the tip is a natural unit of measure since normally this quantity is measured before the experiment begins.

\section{RESULTS}

The behavior of the force in equation (2) can be better understood by plotting $F_{z}$ versus $\eta$ for a variety of ring sizes $\beta$. These plots are rendered in Figure 3. There we see the salient feature that, for all $\beta$, the force-separation curve presents a maximum attraction. Thus, one can set up an experiment whereby, starting from the configuration of tip and substrate (ring's plane) in contact, the tip is pulled away from the substrate while the force is monitored. When the maximum attraction is reached, we stop and record both the maximum force $F_{\max }$ and the separation $\eta_{\max }$. We then keep pulling the tip away until we reach the force $F_{\text {max }} / 2$, and again record the separation at which this new force occurs. 


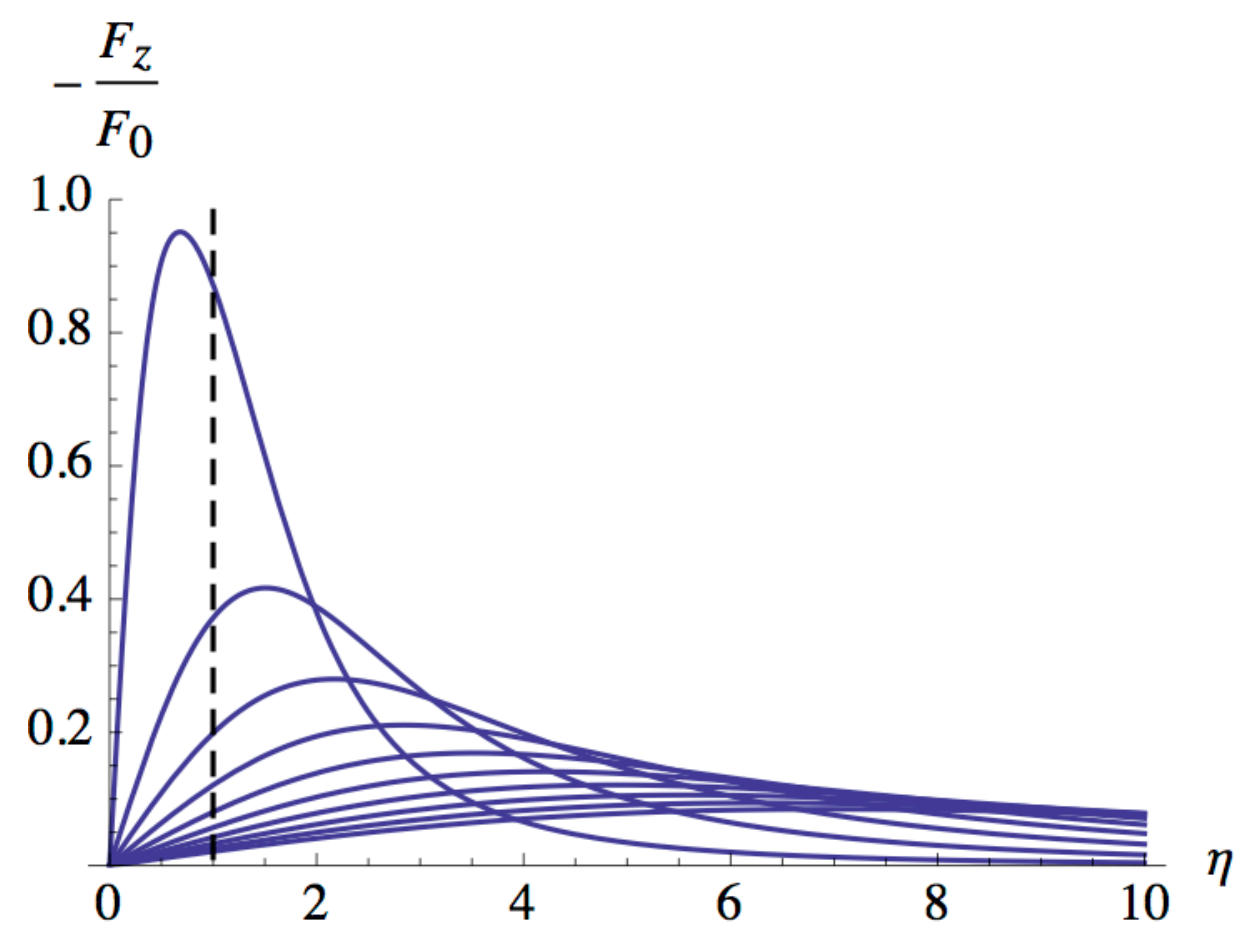

Figure 3. Attractive force-separation curves between tip and ring for various ring radii. The vertical dashed line at $\eta=1$ represents the closest approach: the tip and substrate are in contact then. Thus forces for $0<\eta<1$ are physically unreachable -they would correspond to the tip penetrating the plane of the ring. Curves for which the maximum force occurs in that region correspond to small rings.

For each curve in figure 3 (which corresponds to a different value of $\beta$ ) there is one separation $\eta$-call it $\eta_{\max }$ - for which the force is a maximum. The physical interpretation of $\eta_{\max }$ is the distance at which the AFM tip experiences maximal deflection under experimental conditions. Both the maximum force and its corresponding $\eta_{\max }$ are measured quantities (or can be reduced to those, as discussed below) so the corresponding value of $\beta$ can be determined using them.

We were not able to analytically determine $\eta_{\max }$ as a function of $\beta$. Instead, we compute it numerically. figure 4 presents the results of the numerical study, a 
graph of $\eta_{\max }$ versus $\beta$, found by determining the roots of $\frac{1}{F_{0}} \frac{\partial F_{z}}{\partial \eta}$ for each fixed $\beta$. Values of $\eta_{\max }$ below 1 are physically irrelevant because, as explained, they correspond to the tip going below the sample substrate.

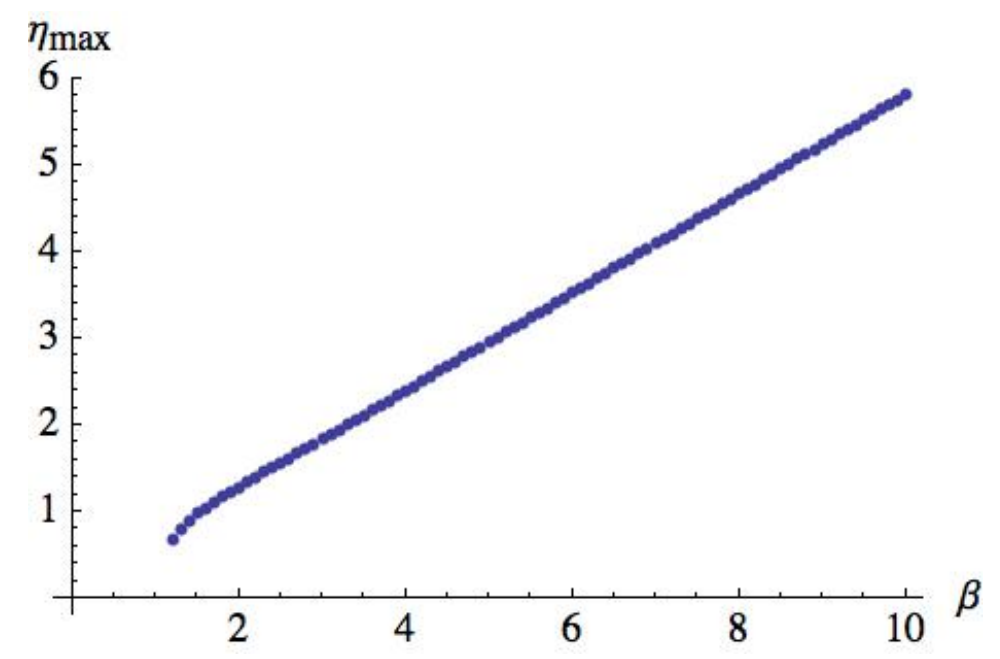

Figure 4. $\eta_{\max }$ versus $\beta$. This is the location of the maxiumum force as a function of the size of the ring. The almost straight line was obtained numerically as detailed in the text.

The relation $\eta_{\max }$ vs $\beta$ turns out to be highly linear in the region of interest, $\beta \geq 1$. A linear approximation with correlation coefficient $R^{2}=0.99996$ is given by

$$
\eta_{\max }=0.5568 \beta+0.1684
$$

Thus given experimental $\eta_{\max }$ the size of the ring $\beta$ is obtained from equation 4. With both $\eta_{\max }$ and $\beta$ in hand, one can determine the charge density, $\lambda$, of the sample ring. Letting $F_{z, \max }$ be the experimentally determined maximum force (in practice, typically in the $\mathrm{nN}$ range), with its corresponding $\eta_{\max }$ value, we then have, as a particular instance of equation 2 : 


$$
F_{z, \text { max }}=-\frac{\lambda^{2}}{\varepsilon_{0}} \frac{\eta_{\max } \beta^{2}}{\sqrt{\beta^{2}+\eta_{\max }^{2}}}\left\{\frac{1-\frac{1}{\beta^{2}+\eta_{\max }^{2}}}{\left[\beta^{2}+\left(\frac{\beta}{\beta^{2}+\eta_{\max }^{2}}\right)^{2}+\left(\eta_{\max }-\frac{\eta_{\max }}{\beta^{2}+\eta_{\max }^{2}}\right)^{2}\right]^{\frac{3}{2}}}\right\} f\left(\mu_{\max }\right)
$$

where we used $F_{0}=\frac{\lambda^{2}}{\varepsilon_{0}}$. Using equation 4 we obtain the charge density $\lambda$ explicitly in terms of known quantities,

$$
\lambda=\sqrt{\frac{F_{z, \max } \varepsilon_{0} \sqrt{\beta^{2}+(0.5568 \beta+0.1684)^{2}}}{(0.5568 \beta+0.1684)^{2} \beta^{2} f\left(\mu_{\max }\right)} \frac{\left[\beta^{2}+\left(\frac{\beta}{\beta^{2}+(0.5568 \beta+0.1684)^{2}}\right)^{2}+\left((0.5568 \beta+0.1684)^{2}-\frac{(0.5568 \beta+0.1684)^{2}}{\beta^{2}+(0.5568 \beta+0.1684)^{2}}\right)^{2}\right]^{\frac{3}{2}}}{\frac{1}{\beta^{2}+(0.5568 \beta+0.1684)^{2}}-1}}
$$

In practice, however, there is a problem because the separation distance $\eta$ is not generally measurable. Indeed, in normal experimental setups the initial separation $\eta_{0}$ is unknown: the AFM only gives the deflection of the cantilever, $\delta$ relative to the body frame of the microscope. The connection between tip-substrate separation distance and tip deflection due to an attractive force is generally $\eta=\eta_{0}-\delta$. In particular, at the location of the maximal force $F_{z, \max }$, the separation distance is $\eta_{\max }=\eta_{0}-\delta_{\max }$, while at half that force we similarly have $\eta_{1 / 2}=\eta_{0}-\delta_{1 / 2}$. Therefore,

$$
\left|\eta_{\max }-\eta_{1 / 2}\right|=\left|\delta_{\max }-\delta_{1 / 2}\right|
$$

which eliminates the unknown quantity $\eta_{0}$, leaving only the experimentally measurable deflections corresponding to the two forces $F_{z, \max }$ and $\frac{1}{2} F_{z, \max }$. Studying $\left|\delta_{\max }-\delta_{1 / 2}\right|$ vs. $\beta$ allows for the determination of $\beta$, which is in turn can be used to obtain $\lambda$ using equation 6 .

Figure 5 shows a graph of $\delta_{1 / 2}-\delta_{\max }$ vs. $\beta$, from which the following linear equation was determined: 


$$
\delta_{1 / 2}-\delta_{\text {max }}=0.8016 \beta+0.0542
$$

with $R^{2}=0.9999$. Thus, by measuring the deflections at maximum force and at half that force the size of the ring can be determined using equation 8 , from which the charge density is calculated using equation 6 .

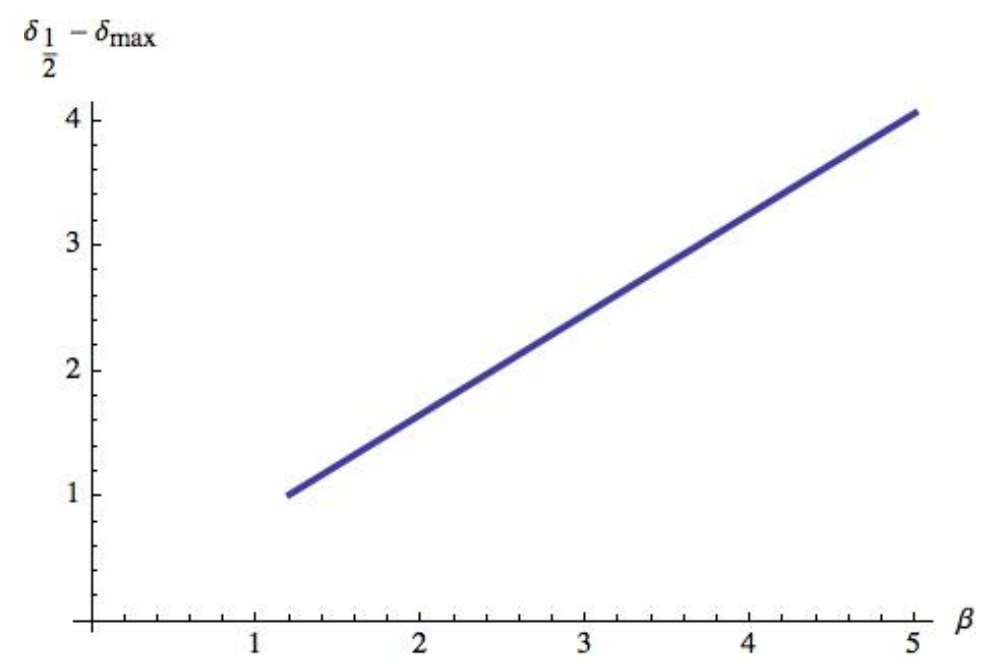

Figure 5. $\delta_{1 / 2}-\delta_{\max }$ vs $\beta$, Relative measurable deflection vs ring size. The result was obtained numerically.

\section{CONCLUSION AND DISCUSSION}

The results obtained in the Results section are significant in that they provide an unambiguous experimental procedure to measure charge content and size using electrostatic AFM.

However, although outliers, there are two outstanding cases to consider before our discussion is complete, for which the method present above cannot be used:

i) There are values of $\beta$ for which theoretically the maximum force occurs only for $\eta<1$, that is, below the sample platform (see figure 3 for an example). 
ii) Moreover, for all $\beta \leq 1$ the force-distance curves do not converge as in Figure 3 and so do not have a maximum. Instead, they have an asymptote in $0<\eta<1$. This is because for rings that are the same size as the sphere or smaller, a possibility opens for the sphere to touch the ring-instead of going straight through it, as for $\beta>1$-causing the distance between the charges in the two objects to go to zero, and the corresponding Coulombic force to diverge. Thus, for $\beta \rightarrow 0$ the sphere would touch the tip right at $\eta=1$, which is where the asymptote will occur. Likewise, for $\beta=1$ (a ring whose radius is exactly the same as that of the sphere), the asymptote would occur at $\eta=0$, when the sphere center coincides with that of the ring.

In both these cases, there is no experimentally available maximum force-either relatively, because $F_{\max }$ is in the $\eta<1$ region that simply cannot be measured, or absolutely, since the force grows asymptotically in that same region. In both these cases, a modified method must be used, since the maximal measurable force occurs at $\eta=1$. Unlike before, though, one need not measure forces away from the platform. Once the (relatively) maximum force has been measured at the sample platform, one can immediately obtain $\beta$ using equation 2 and finally find $\lambda$ using equation 6.

\section{ACKNOWLEDGMENT}

Work supported by the National Science Foundation through grant \#CHE-1508085.

\section{REFERENCES}

${ }^{1}$ M. Peplow, Nature 525 (2015) 18-21

2 Z. Zhang, S. Witham, E. Alexov, On the role of electrostatics on protein-protein interactions. Physical Biology 8 (2011) 035001. doi:10.1088/1478-3975/8/3/035001.

${ }^{3}$ Y. Wang, N. Yang, and J.-L. Zhu, Aharonov-Bohm phase operations on a double-barrier nanoring charge qubit Phys. Rev. B 74 (2006) 035432 
${ }^{4}$ M. Dunaevskiy, P. Alekseev, P. Girard, A. Lashkul, E. Lahderanta, A. Titkov, Analysis of the lateral resolution of electrostatic force gradient microscopy, Journal of Applied Physics 112, 064112 (2012), DOI:http://dx.doi.org/10.1063/1.4752430.

${ }^{5}$ B. M. Law and F. Rieutord, Electrostatic forces in atomic force microscopy Phys. Rev. B 66 (2002) 035402

${ }^{6}$ S.F. Lyuksyutov, R.A. Vaia, P.B. Paramonov, S. Juhl, L. Waterhouse, R.M. Ralich, G. Sigalov. E. Sancaktar, Electrostatic nanolithography in polymers using atomic force microscopy, Nature Materials 2 (2003) 468-472 doi:10.1038/nmat926. This reference used spheres to model the apex of pyramidal tips for electrostatic applications.

${ }^{7}$ A. Boularas, F. Baudoin, C. Villeneuve-Faure, S. Clain, G. Teyssedre, Multi-dimensional modelling of electrostatic force distance curve over dielectric surface: Influence of tip geometry and correlation with experiment, Journal of Applied Physics 116 (2014) 084106 doi: 10.1063/1.4894147. This reference shows the dominant effect of the spherical tip on the total force mostly at tip-sample separations smaller than the tip apex's size.

8 The company Nanoandmore GmbH has a good inventory of spherical tips on cantilevers. See for example model CP-FM-Au-A-5, a 1 micron gold spherical tip at http://www.nanoandmore.com/AFM-Probe-CP-FM-Au.html

${ }^{9}$ A. Sadeghi,A. Baratoff, S. Goedecker, Electrostatic interactions with dielectric samples in scanning probe microscopies, Physical Review B 88 (2013) 035436, DOI:

10.1103/PhysRevB.88.035436

10 J.D. Jackson, Classical Electrodynamics, $3^{\text {rd }}$ Edition, section 2.2, John Wiley \& Sons, New York, 1999

${ }^{11}$ A. Bonanno, M. Camarca, P. Sapia, Magnetic interactions and the method

of images, Eur. J. Phys. 32 (2011) 849-866 doi:10.1088/0143-0807/32/4/001

12 L. Gao, D. Zhao, Electric force between two charged Coaxial rings, Far East Journal of Mathematical Sciences (FJMS) 70 (2012) 135-143

${ }^{13}$ F.R. Zypman, Off-axis electric field of a ring of charge, Am. J. Phys. 74 (2006) 295, http://dx.doi.org/10.1119/1.2149869

${ }^{14}$ M. Abramowitz and I.J. Stegun, Handbook of Mathematical Functions, National Bureau of Standards, AMS 55, $2^{\text {nd }}$ printing, 1964 


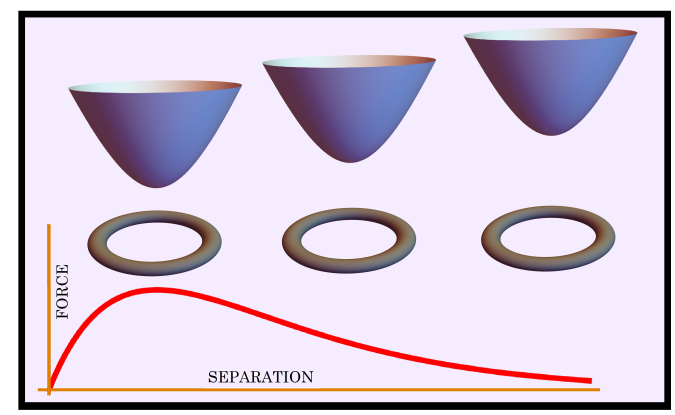

GRAPHICAL ABSTRACT 